POS $\quad$ PROCEEDINGS

\title{
Finding AGN in Deep X-ray Flux States with Swift
}

\section{Dirk Grupe}

Department of Earth and Space Science, Morehead State University, 235 Martindale Dr., Morehead, KY 40351, USA E-mail: d.grupe@moreheadstate.edu

\section{S. Komossa*}

Max-Planck-Institut für Radioastronomie, Auf dem Hügel 69, 53121 Bonn, Germany

E-mail: skomossa@mpifr-bonn.mpg.de

\section{Mason Bush, Chelsea Pruett, Sonny Ernst, Taylor Barber, Jen Carter}

Department of Earth and Space Science, Morehead State University, 235 Martindale Dr., Morehead, KY 40351, USA; Rowan County Senior High School, Morehead, KY, 40351, KY, USA

\section{Norbert Schartel, Pedro Rodriguez, \& Maria Santos-Lleó}

XMM-Newton Science Operations Centre, ESA, Villafranca del Castillo, Apartado 78, 28691

Villanueva de la Cañada, Spain

We report on our ongoing project of finding Active Galactic Nuclei (AGN) that go into deep $\mathrm{X}$-ray flux states detected by Swift. Swift is performing an extensive study on the flux and spectral variability of AGN using Guest Investigator and team fill-in programs followed by triggering XMM-Newton for deeper follow-up observations. So far this program has been very successful and has led to a number of XMM-Newton follow up observations, including Mkn 335, PG 0844+349, and RX J2340-5329. Recent analysis of new Swift AGN observations reveal several AGN went into a very low X-ray flux state, particularly Narrow-Line Seyfert 1 galaxies. One of these is RX J2317-4422, which dropped by a factor of about 60 when compared to the ROSAT All-Sky Survey.

Swift: 10 Years of Discovery

2-5 December 2014

La Sapienza University, Rome, Italy

\footnotetext{
${ }^{*}$ Speaker.
} 


\section{Introduction}

The Swift mission [1] has revolutionized the ability to monitor and study Active Galactic $\mathrm{Nu}$ clei (AGN). With its flexible scheduling and multi-wavelength capacity, Swift has enabled observers to quickly react to changes discovered in the fluxes of AGN. AGN often exhibit rapid and dramatic flux and spectral changes in X-rays. These changes can be associated with absorption, reflection, or dramatic changes in the accretion rate. Some of the most dramatic X-ray flux and spectral changes have been observed in Narrow Line Seyfert 1 galaxies (NLS1s). This class of AGN is characterized by relatively small black hole masses and high accretion rates relative to the Eddington accretion rate. The most extreme of these AGN is the NLS1 WPVS 007 which was bright in the X-ray sky during the ROSAT All-Sky Survey (RASS) in 1990. However, when observed a few years later with ROSAT its X-ray flux had dropped by a factor of 400 [2]. During the RASS it WPVS 007 displayed the softest X-ray spectrum of all bright X-ray detected AGN. FUSE observation in 2003 revealed strong absorption troughs in the UV spectrum of WPVS 007, which are unseen in lowluminosity AGN. These deep absorption troughs are seen in Broad Absorption line Quasars (BAL QSOs) and are the results of strong outflows. WPVS 007 represents a link between BAL QSOs and NLS1s.

This research compares AGN X-ray fluxes found in the ROSAT All-Sky Survey with measurements from recent Swift observations. A decrease in the X-ray flux of an AGN can result from absorption by gas, reflection on the accretion disk, or a change in the accretion rate. Out of 80 AGN analyzed, six were found in a low X-ray flux state. A $1 \mathrm{ks}$ Swift observation of each AGN confirmed our findings. One object, RX J2317-4422, was found to have decreased in X-ray flux by a factor of about 60 [3]. A follow up observation of RX J2317.8-4422 with XMM-Newton reveals an usually soft X-ray spectrum.

\section{Sample Selection and Swift}

The Active Galactic Nuclei are selected from the ROSAT All-Sky Survey (RASS) Bright Source Catalogue by [4]. The total sample size is 400 AGN, many of which have already been observed by Swift multiple times [5]. Between 2014 April and September Swift observed 80 of these AGN.

The Space Science Center at Morehead State University in Eastern Kentucky has an apprenticeship program that allows local senior high school students to participate in active research projects. In Fall 2014 four high school students from the Rowan County Senior High School participated in this program. Each student was given 20 AGN to check for significant X-ray flux variability.

\section{Description of the Data Analysis}

To compare the Swift XRT count rates with those obtained during the RASS, dividing the RASS count rate by 4 is an appropriate estimate of the expected count rate. Students compared the expected Swift XRT count rate with the measured Swift XRT count rate. The XRT count rates were obtained by using the Swift XRT analysis web-interface at the University of Leicester Swift website 


\begin{tabular}{lccc}
\hline Name & $\begin{array}{c}\text { Expected Swift } \\
\text { Count Rate }\end{array}$ & $\begin{array}{c}\text { Actual Swift } \\
\text { Count Rate }\end{array}$ & $\begin{array}{c}\text { Factor } \\
\text { Change }\end{array}$ \\
\hline RX J2317.8-4422 & 0.1775 & 0.010 & 17.8 \\
RX J2216.8-4451 & 0.3250 & 0.0325 & 10 \\
PKS 2310-322 & 0.085 & 0.010 & 8.5 \\
RX J1007.1+2203 & 0.155 & 0.025 & 6.2 \\
RX J1117.1+6522 & 0.1375 & 0.025 & 5.5 \\
MS 0117-28 & 0.135 & 0.025 & 5.4 \\
\hline
\end{tabular}

Table 1: List of AGN which have been found to show a decrease in their X-ray flux obtained by Swift compared with the ROSAT All-Sky Survey (RASS). The first column lists the source names, the second the expected Swift XRT count rate estimated from the RASS count rate, the actually measured XRT count rate, and the last column lists the factors between the estimated and measured count rates.

$[6,7]$ (http://www.swift.ac.uk/user_objects/;). Students were task to find AGN which exhibited a change in their count rate by factors of 5 or greater. Out of the sample of 80 AGN, 6 displayed a count rate that was at least a factor of 5 below the expected value. The 6 AGN that exhibited shuch a large change in their count rate are listed in Table 1. To confirm the X-ray low states, short $1 \mathrm{ks} \mathrm{Swift} \mathrm{ToO}$ observations were requested. Chelsea Pruett became the first high school student ever requesting and obtaining a ToO observation with Swift. All ToO observations confirmed the X-ray low state. One AGN turned out to be very interesting: RX J2317.8-4422, which displayed a decrease in its X-ray flux by a factor of 20 .

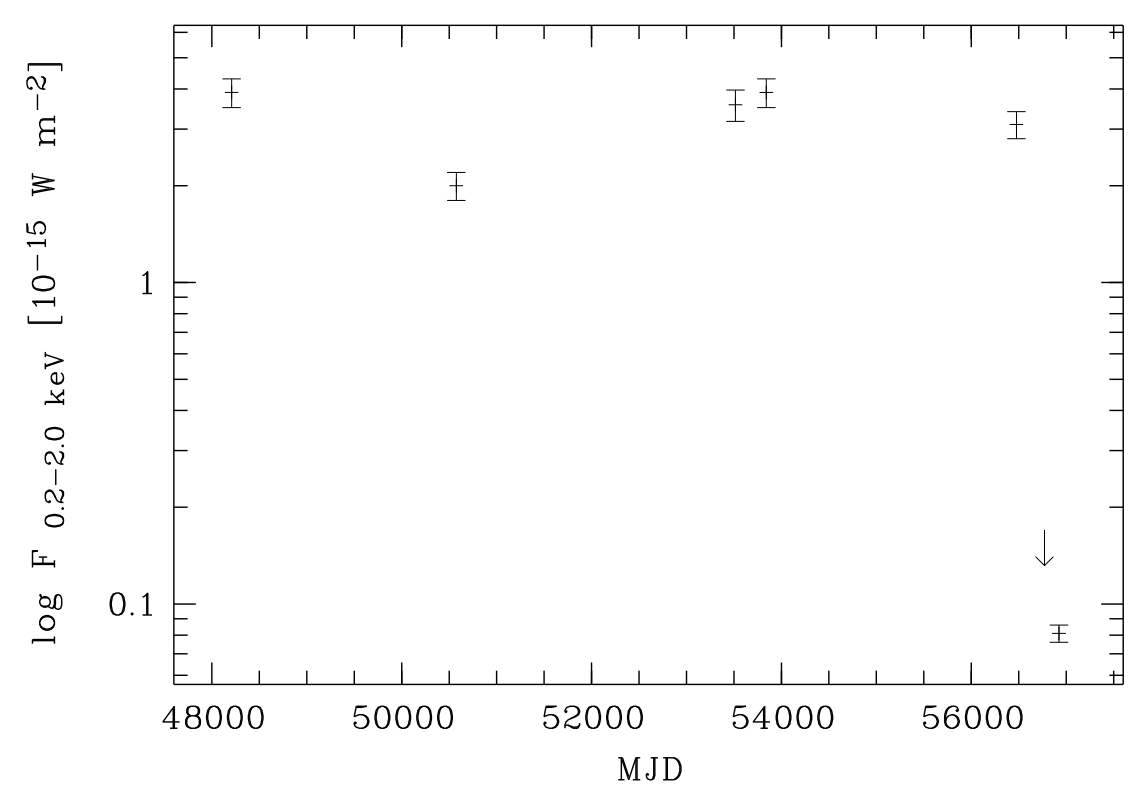

Figure 1: Long-term 0.2 - $2.0 \mathrm{keV}$ light curve of RX J2317.8-4422. The first data point is the X-ray flux during the RASS and the second data point was derived from a ROSAT HRI observation (see [8]). All other data points were derived from Swift observations starting in 2007 [5]. 


\section{The NLS1 RX J2317.8-4422}

RX J2317.8-4422 (RA-2000: 2317 49.9; Dec-2000: -44 22 28; z=0.132) is a Narrow-Line Seyfert 1 galaxy that has exhibited X-ray variability in the past with factors up to $7[8,5]$. Swift observed this NLS1 in April 2014 and obtained a $3 \sigma$ upper limit of $1.76 \times 10^{-16} \mathrm{~W} \mathrm{~m}^{-2}$, which means a drop by a factor of more than 20 compared with the previous observation in August 2013. Figure 1 displays the long-term 0.2-2.0 keV light curve of RX J2317.8-4422. We triggered a Swift ToO observation in September 2014 to confirm this X-ray flux low state and detected the AGN at a level of $8 \times 10^{-17} \mathrm{~W} \mathrm{~m}^{-2}$.

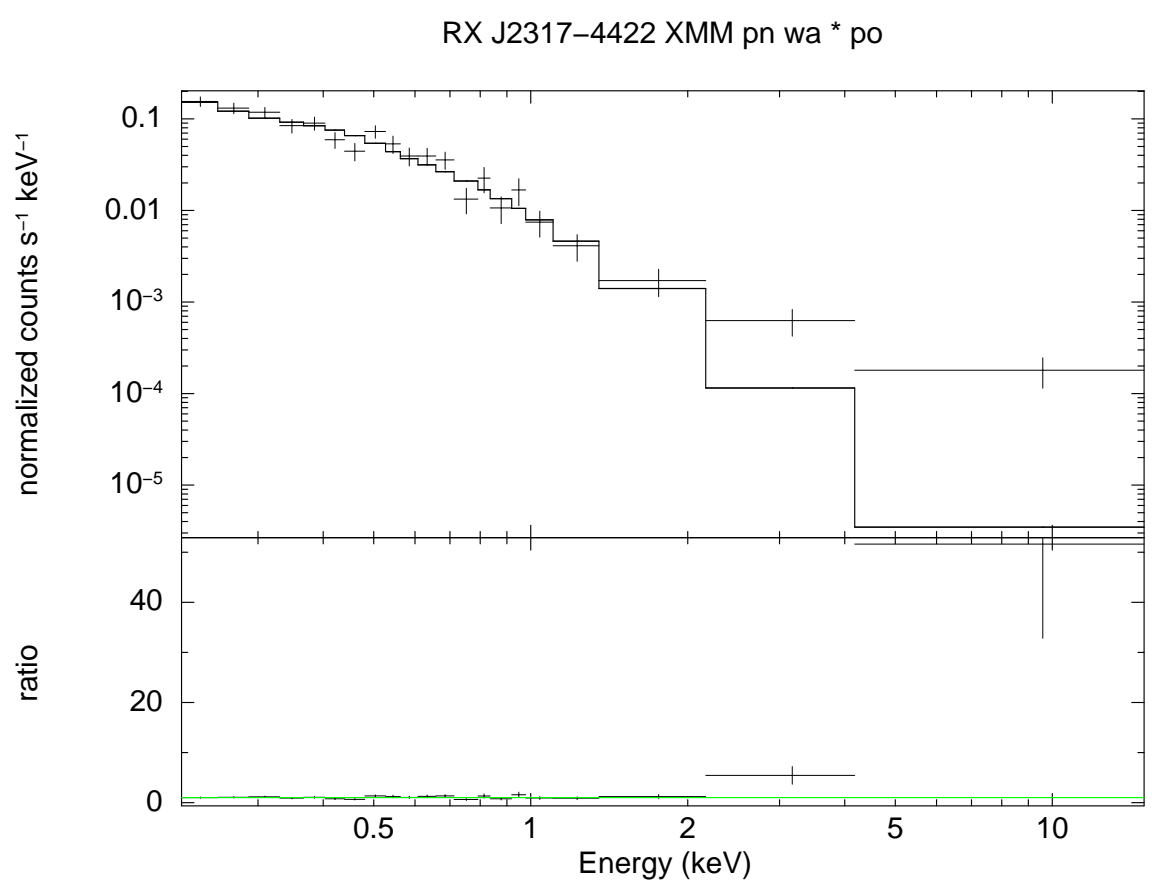

Figure 2: $0.2-12 \mathrm{keV}$ X-ray spectrum of RX J2317.8-4422 obtained by the EPIC pn onboard XMM-Newton on 2014-October-29 fitted with a single power law model with $\alpha_{\mathrm{x}}=2.81$.

The extreme X-ray flux change of RX J2317.8-4422 made it a valid target for an XMMNewton follow up program of AGN in deep minimum X-ray flux states. We obtained an observation with XMM-Newton for $13 \mathrm{ks}$ on 2014-October 29. The X-ray spectrum obtained with the EPIC pn shown in Figure 2 shows a very soft spectrum that can be modeled by a single power law model with an X-ray spectral slope of $\alpha_{\mathrm{x}}=2.81 \pm 0.21$ with an absoption column density fixed to the Galactic value $N_{\mathrm{H}}=1.07 \times 10^{20} \mathrm{~cm}^{-2}\left(1.07 \times 10^{26} \mathrm{~m}^{-2}\right)$. There is a hard X-ray component in the spectrum. To better understand the cause of this X-ray spectrum a second XMM-Newton observation was performed. A 100ks observation was performed by XMM-Newton on 2014-November-17 simultaneously with Swift and NuStar. The second XMM-Newton observation showed that the X-ray flux of RX J2317.8-4422 had decayed even more and was found at a level of $(6.0 \pm 0.6) \times 10^{-17}$ $\mathrm{W} \mathrm{m}^{-2}$. Figure 3 compares the spectral energy distributions of RX J2317.8-4422 during the RASS (large squares) and the XMM-Newton observation on October 29. 


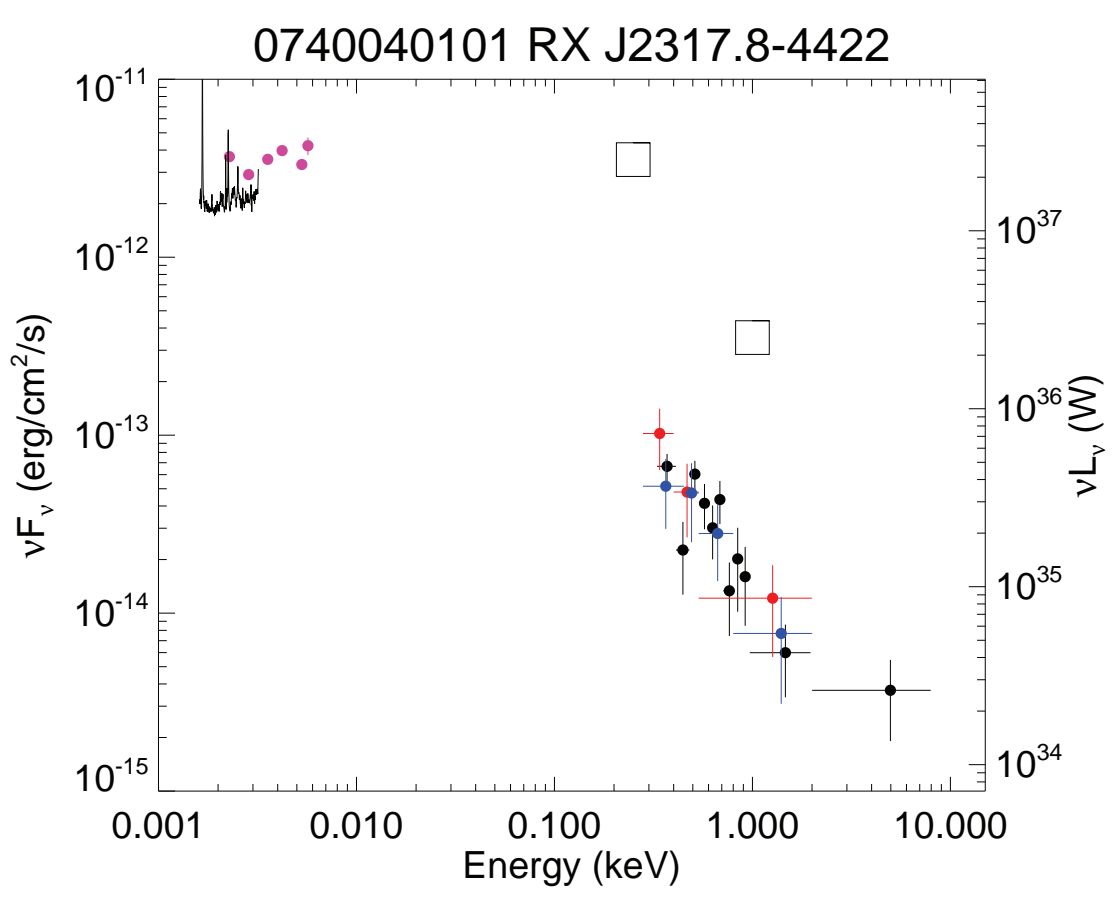

Figure 3: Spectral Energy Distribution of RX J2317-4422. The large squares display the X-ray data during the RASS. The optical/UV data points were derived from the XMM-Newton OM, and the X-ray data were obtained from the EPIC pn and MOS detectors. The optical spectrum was taken in 1995 with the ESO $1.52 \mathrm{~m}$ telescope in La Silla/Chile $[9,10]$.

\section{Conclusions}

Results of finding AGN in deep minimum states have again shown that Swift has become a game changer in finding these unusual AGN. Swift is not only able to survey large samples of AGN very efficiently, it also allows fast follow up observations to verify the state of an AGN. Our Swift fill-in program of observing AGN is ongoing and is expected to find more AGN with high $\mathrm{X}$-ray variability in the future. The goal of the fillin program is to understand the changes in the spectral energy distributions of AGN. We can conclude that these AGN in deep minimum states under extreme conditions. Our current understanding of the dramatic changes in the X-ray flux and spectra is that this can be caused by absorption or X-ray reflection [11, 12]. A preliminary analysis of the XMM-Newton observation of RX J2317.8-4422 from November 2014 displays very soft X-ray spectrum, similar to that of WPVS 007 during the RASS. Is RX J2317.8-4422 maybe the next link between NLS1s and BAL QSOs? We are currently working on the publication of the Swift, NuStar, and XMM-Newton observations of RX J2317.8-4422. 


\section{References}

[1] N. Gehrels, G. Chincarini, P. Giommi, et al., The Swift Gamma-Ray Burst Mission, ApJ 611 (2004) 1005

[2] D. Grupe, et al., Discovery of an ultrasoft transient ROSAT AGN: WPVS 007, Astron. Astrophys. 300 (1995) L21

[3] D. Grupe, et al., Discovery of the Narrow-Line Seyfert 1 Galaxy RX J2317.8-4422 in a very deep $X$-ray Low State by Swift, ATEL $\mathbf{6 6 0 7}$ (2014)

[4] A. Schwope, et al., The ROSAT Bright Survey: II. Catalogue of all high-galactic latitude RASS sources with PSPC countrate CR $>0.2 s^{-1}$, AN 321 (2000) 1

[5] D. Grupe, S. Komossa, K.M., Leighly, \& K.L. Page, The Simultaneous Optical-to-X-Ray Spectral Energy Distribution of Soft X-Ray Selected Active Galactic Nuclei Observed by Swift, ApJS 187 (2010) 64

[6] P. Evans, et al., An online repository of Swift/XRT light curves of gamma-ray bursts, A\&A 469 (2007) 379

[7] P. Evans, et al., Methods and results of an automatic analysis of a complete sample of Swift-XRT observations of GRBs, MNRAS 397 (2009) 1177

[8] D. Grupe, H.-C. Thomas, \& K. Beuermann, X-ray variability in a complete sample of Soft X-ray selected AGN, A\&A 367 (2001) 470

[9] D. Grupe, et al., New bright soft X-ray selected ROSAT AGN. II. Optical emission line properties, $A \& A 350$ (1999) 805

[10] D. Grupe, B.J. Wills, K.M. Leighly, \& H. Meusinger, A Complete Sample of Soft X-Ray-Selected AGNs. I. The Data, AJ 127 (2004) 156

[11] D. Grupe, et al., A Remarkable Long-term Light Curve and Deep, Low-state Spectroscopy: Swift and XMM-Newton Monitoring of the NLS1 Galaxy Mkn 335, ApJS 199 (2012) 28

[12] L.C. Gallo, et al., Suzaku observations of Mrk 335: confronting partial covering and relativistic reflection, MNRAS 446 (2015) 633

\section{Acknowledgments}

This work made use of data supplied by the UK Swift Science Data Centre at the University of Leicester. The work is based on observations obtained with XMM-Newton, an ESA science mission with instruments and contributions directly funded by ESA Member States and NASA. We also want to thank Neil gehrels and the Swift Mission Operation Center to make every effort to get our ToO requests scheduled. 